\title{
Introducing the Concept of Internet of Digital Reality - Part I
}

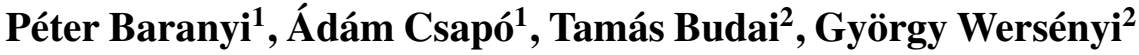

1 Dept. of Computer Science, Széchenyi István University

Egyetem tér 1, Győr, Hungary

baranyi.peter@sze.hu, csapo.adam@sze.hu

2 Dept. of Telecommunications, Széchenyi István University

Egyetem tér 1, Győr, Hungary

budai.tamas@sze.hu,wersenyi@sze.hu

\begin{abstract}
With the growing pervasiveness of Virtual Reality, Augmented Reality, Mixed Reality and Digital Twins combined with Artificial Intelligence, 5G networks and the omni-present $2 D$ Web, we are entering into a new era, characterized by a multi-modal entangled combination of previously disparate realms of IT with human and social cognitive systems. This process of entanglement is arguably leading to a new, qualitatively different kind of reality, in which the borders between the physical and digital world, as well as digital representations and simulations thereof are becoming increasingly fuzzy. Based on these developments, the paper re-interprets some well-known concepts - including virtual reality, augmented reality, mixed reality, as well as virtual / digital simulations and virtual / digital twins. Based on these new interpretations, the paper introduces the higher-level structures of Digital Reality $(D R)$ and Internet of Digital Reality (IoD). It is argued that these structures can lead to a better understanding of the new possibilities afforded to humanity by pervasive digital technologies.
\end{abstract}

Keywords: Digital Reality; Future Internet; Internet of Things; Internet of Everything; Internet of Digital Reality

\section{Introduction}

With its rapid evolution in the past decades, modern information technology has arguably led to a fundamental re-shaping of the landscape of human cognition, for better or worse [1, 2]. However, regardless of the ways in which individual capabilities, behaviors and socio-cognitive structures are impacted, digitization is a phenomenon that is not only here to stay with us, but can also be expected to deepen in its pervasiveness within all aspects of the everyday human experience. This development has led to a variety of new conceptualizations in terms of the coevolution of humans, machines, and computer networks - including human-machine 
entanglement, cognitive merging / co-evolution, singularity, and even post-humanism. All of these concepts highlight the general idea that the border between humans and technology is becoming increasingly fuzzy $[3,4,5,6]$, and all of them are deeply investigated under the scientific discipline of Cognitive Infocommunications (CogInfoCom) [6, 7].

This paper is based on this realization that humanity has reached an inflection point in its social and technological evolution, and details how the continued deepening of human-ICT entanglement can be expected to lead to a qualitatively new kind of reality, referred to as the digital reality. In turn, the communication, management and harmonization aspects of different segments of this reality are covered by the concept of Internet of Digital Reality (IoD).

Note that the term 'digital reality' is not entirely new - Deloitte Consulting LLP and the Consumer Technology Association have introduced 'digital reality' as a trademarked term to refer to "technologies and capabilities that inhere in $A R, V R, M R, 360^{\circ}$ video, and the immersive experience, enabling simulation of reality in various ways" as described in $[8,9,10]$. In this paper, our goal is to add more context and a wider perspective to this term - focusing not only on the technology (how information is visualized) but also highlighting artificial intelligence related, capability-oriented and social cognition aspects. We also consider the ramifications of the concept of digital reality in the emergence of a new kind of interconnectedness, referred to as the Internet of Digital Reality (IoD). In a separate paper (Part II), we provide a more in-depth discussion on the infrastructural background of IoD [11].

\section{Key Concepts}

In order to motivate and clearly delineate Digital Reality as a concept, we first take a look at some related technological and social concepts. Our exploration involves casting new light on some commonly held assumptions about the fields of virtual reality, augmented reality, mixed reality and others. Based on these discussions, a new perspective emerges which helps us define Digital Reality at the end of this section. Later in the paper, the term 'Internet of Digital Reality' is further explored.

\subsection{The Concept of Reality}

First of all, it is important to clarify what we mean by the term 'reality'. In this paper, we use the working definition of reality as being a set of conceptions and perceptions that form an integrated unit of comprehension, and create an understanding of what is possible, desirable and actual.

As we will see later, this definition covers quite many details of human experience at any given time and in any given historic moment. By 'integrated unit of comprehension', we mean that even if a reality is comprised of many different components, 
at a higher level (the level of the reality), those components together create a new meaning which does not exist at the individual level of the components. By highlighting what is possible, desirable and actual, we mean that a reality can shape our experience at a basic level.

The definition also highlights the fact that as long as a set of possibilities, desires or actual manifestations of the world are mutually exclusive, or alternatively, as long as a set of possiblities, desires or actual manifestations are focused on entities that cannot have a meaningful influence over one another, then we can speak of different realities. For example, the files on one person's computer and the files on another person's computer belong to different realities - at least in a technical sense - as long as the two people are strangers to each other and live their lives in complete independence of one another. At the same time, the files on one person's computer and the same person's filing cabinet could likely belong to the same reality - the given person's reality - regardless of the former existing in digital form, and the latter in physical form. These examples show that the concept of reality is orthogonal to the dimensions of physical / digital, and real / virtual - an idea that may seem counterintuitive at first, given the colloquial associations created by words such as 'virtual reality', generally used in to cover computer-generated 3D applications.

Expressed differently, the fact that virtual reality, augmented reality and physical reality are treated as different concepts today (based on the medium through which they are primarily accessed) merely shows that the technology behind them has not (yet) achieved a level of pervasiveness based on which we are able to access, manipulate and organize the same information regardless of the medium we are using. If this were the case, virtual and physical reality would significantly overlap.

\subsection{Layers of virtuality}

In its colloquial sense, the word 'virtual' is often used to refer to something that is not real, but is a manifestation that somehow resembles a real counterpart. Although widespread associations behind the term 'virtual' most often have to do with digital manifestations that resemble physical objects, we consider this interpretation to be limited, especially from the perspective of the definition of reality provided in Section 2.1. For this reason, we consider any manifestation to be 'virtual' that has a referential aspect, regardless of whether that manifestation appears purely in someone's imagination, or in a specific physical or digital solution, and regardless of whether it points to a real (physical) or a purely imaginary concept, or to a specific, concrete object (i.e. an instantiation of a concept).

Going deeper into this term, we note that:

- the term 'virtual' is not an absolute term and (contrary to the commonly held viewpoint) is not even necessarily digital - e.g. both a card game on a table or a 3D digital game can be thought of as virtual, depending on what realities the cards and the games themselves refer to. A tangible user 
interface (a TUI, which is completely physical) can be considered as a virtual tool, provided that it refers to (controls and / or is updated in synchrony with) a 'backend system' (whether digital or physical - though mostly digital in the original formulation of TUIs $[12,13])$. Similarly, open-air museums of traditional villages (e.g. Skansen) can be considered as 'virtual' (despite being physical and not even referring to anything digital), as they represent an architecture and way of life from a bygone era (to visit Skansen is to visit a location that resembles a typical village from the past). Based on the above, the term 'virtual' can be conceived of as a relative term that focuses on an aptly defined border between artificial and natural, or some constructed reality and a base reality;

- the term 'virtual' does not necessarily refer to visual (or visual only) manifestations. While most people associate the term 'virtual' (as in 'virtual reality' described below) with visual inputs, resemblance to the referent counterpart will in general depend on the context - whether that counterpart has a visual appearance (either in 2D or 3D), or if it has affordances in other modalities, or if it is purely an abstract concept. Note that in some interpretations, such as in the framework of embodied cognition, nothing exists that is purely abstract (i.e., divorced from the spatial experience / spatial interactions of the human mind-body) [14], we can at least assume that it is possible to distinguish among concepts that are closer to or more removed from physical reality $[15,16]$.

In summary, it is possible to characterize both physical and digital objects that are perceived by humans as being virtual to a different extent, depending on their proximity to the reality they refer to. A digital representation of a physical object that in its specific form does not exist in reality can seem more virtual (less real) than a digital representation of an object (an instantiation of the concept) that specifically really exists in physical reality. By contrast, physical objects (like paper money) can often seem even less virtual, though in a sense they are absolutely virtual in that they are an abstraction that correlates with someone's services to society as recognized by that society.

\subsection{Characterizing Realities by Degree of Physicality: Virtual, Aug- mented and Mixed Realities}

According to the commonly held view, virtual reality (VR) refers to a higher-level arrangement of computer-generated and visually displayed objects that resemble objects in physical reality.

However, as described above, the concepts 'reality' and 'virtual' are orthogonal to the medium or specific technology that is being used. Therefore, in much the same way as the term 'virtual', 'virtual reality' can be conceived of as any artificially constructed environment (whether physical or digital) that contains virtual objects. It 
is important to point out that VR can be similar to or completely different from the real physical world, and can be experienced using both physical or digital tools. In other words, the sign / symbol and the referent (i.e., the referred-to) 'components' of a virtual reality can be either physical or digital, in any combination.

Today, VR is typically implemented on digital devices (usually 3D displays) and most recently it has become associated with 3D glasses, which provide an immersive experience into a complete virtual environment generated by computers. In this regard, VR is generally considered as a set of 3D visualizations describing any artificial or real world (although other perceptual modalities like auditory, haptic, somatosensory and olfactory cues can also be relevant. However, the fact alone that an environment is 2D (and not 3D), not primarily visual (as opposed to visual), augmentative (in the sense that it does not 'shut out' the physical environment as opposed to being completely immersive), or physical (as opposed to digital) - indeed, any combination of the above - does not mean that the environment cannot be artificially constructed and that it cannot refer to other concepts or objects. Further, it has no bearing on what kind of 'mixture' it represents between the physical and the digital - one can be standing in an insulated room wearing a completely immersive headset, and yet one's thoughts and feelings can refer to concepts and objects in any substrate, both physical and digital - hence refer to the same 'reality'. It can even be argued that VR is a novel kind of infocommunication tool, with direct consequences to everyday life [17]. As we will see, this is an important feature of all computer-generated realities, especially when considering their capability of referring to realities at different levels of virtuality, as described later in Section 2.4.

Augmented reality (AR) most often refers to a computer-generated reality that involves virtual objects presented on a visual field inside a physical environment. Note, that as in the case of VR, an augmented reality need not necessarily be generated by a computer. However, it is clear that the key distinguishing feature of AR as opposed to VR is that the user is primarily located in a 'physical base reality', which is somehow augmented.

In general, the physical objects in the user's environment can serve as fixtures that can accommodate various data representations - e.g. a large dashboard or video display might be projected onto an empty vertical surface like a wall, whereas a more structured and single-purpose display like a clock might be projected onto a smaller, flat surface like the top of a night stand. Since the human body is also a part of physical reality, we consider any augmentation of the human body (e.g. with wearable displays or bionic devices integrated into the body) as a part of augmented reality.

Because of its supplementary nature to physical reality, AR is often also referred as extended reality (XR). Also somewhat related to AR is the concept of mixed reality (MR), which is defined as an environment in which "real world and virtual world objects are presented together within a single display", and that falls "anywhere between the extrema of the Virtuality Continuum" [18] - a hypothetical continuum between the two extremes of the purely physical and the purely virtual, 
encompassing both augmented reality and augmented virtuality. In the terminology of the virtuality continuum, VR is regarded as a singular 'limiting case' in which everything is purely virtual. All other realities are mixed by necessity. However, if the notions of reality and virtuality are considered in the sense described in Sections 2.1 and 2.2, the notion of virtuality hinges not on a specific substrate (digital or physical) and even a single reality can reach across the border between digital and physical. This is well demonstrated by the fact that the terms virtual reality and augmented reality are often used side-by-side or interchangeably [19] ${ }^{1}$.

As described in the following subsection, computer-generated realities can be characterized not only based on the levels of virtuality in which and with which they operate, but also based on the qualities of the 'referent realities' that are being referred to by the objects and processes within those computer-generated realities.

\subsection{Linking Together Realities via Representation and Simulation}

Computer-generated realities derive their strong potential not simply from their ability to present objects that are reminiscent of physical objects in some abstract or entertainment-driven sense, nor from their ability to overlay information about imaginary entities on physical objects. By the same token, their power isn't necessarily derived from the way in which they are merged with our 'physical base reality' whether they appear on portable, see-through displays or on the screen of a desktop computer.

Rather, computer-generated realities are immensely powerful due to their ability to "speak" to humans not just about concepts, but also about objects that exist in different realities, using a varied language that includes both familiar objects and abstract representations. In this subsection, the referential capabilities of computergenerated realities (i.e. their 'aboutness' with respect to other realities) are described via the concepts of simulation and twin representation.

A virtual simulation (VS) is a simulation of the 3D motion and changes in the geometrical 3D representation of an object (whether physical or abstract) in a computergenerated or in a physical environment. Note that although in most cases, virtual simulations involve depicting the appearance and motions of a physically existing object in a computer-generated world, the definition of VS nevertheless allows other combinations between the physical and non-physical to refer to each other, as long as both the sign / symbol and the referent have temporal geometric properties. This is the reason why a purely abstract process, like the training process of a deep neural network, can also be the subject of a virtual simulation, if the goal is to show some (simplified, aggregated) aspects of the training process to e.g. a group of students or experts using graphical (geometric) concepts. In this sense, any graphical model that has a temporal dimension can be considered as a virtual simulation, when the temporal dimension is being explored. In any case, a VS by definition focuses on

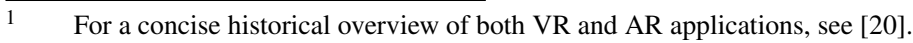


the visualization of geometrical properties and / or motion in 3D.

In some sense more generally, and in another more specifically, a digital simulation (DS) is a computer-generated simulation of a temporal process (whether a real, physical, purely imagined or digital process). In the case of $\mathrm{DS}$, the $3 \mathrm{D}$ virtual (geometric) representation of various objects is not in primary focus (or, in the extreme case, has no meaning at all), since the purpose is to represent processes, which by necessity appear as changes through time, using digital technologies. The concept of DS therefore includes components that may abstract away from the 'highfidelity visual replica' viewpoint of VR and can be considered as a digitized version of a given physical process, either actually taking place or imagined. In some cases, even the potential future states of the system can be estimated (or simulated) and presented to users, thereby providing a 'multi-state' representation. Returning to the previous example of simulating deep neural networks, if the goal is not to present the workings of the model to humans, but rather to run it in order to obtain viable estimates for certain hyper-parameters (which are then later used as a part of the 'real' training process), then this hyper-parameter search can be considered as a digital simulation.

Note that both VS and DS can be used to simulate purely mental or digital phenomena (i.e. objects and processes can refer to cognitive objects and cognitive representations of processes, or to processes that are already being run on computers). Due to its process-based quality, in an extreme case, DS does not necessitate the use of any specific 3D representation from the physical world - whereas in the case of VS, the $3 \mathrm{D}$ representations are by necessity object-based as they involve the transmission of geometrical properties.

Moving further on in our exploration of bridges between physical and mental / computer-generated realities, it is useful to consider the concepts of virtual twins and digital twins.

A virtual twin (VT) is an object that refers to another (referent) object in a different reality, and whose geometric appearance is continuously updated to reflect the geometric appearance of that referent object. VT primarily refers to a high fidelity geometric copy with a photo-realistic visualisation of various physical features such as color, materials / textures etc. in VR. The 3D motion of a VT can also be synchronized in real time with that of its counterpart, in cases where the VT is used to control the real object, or the real object is used to control the VT (control / monitoring). In practice, the motion of the 3D representation of the VT is based on a combination of information from simulations (VS) and the real object. In general, we can say that if there are two objects, one being a real physical object, and the other one represented in VR, and the two are purposely similar in visual appearance and in 3D motion, then the application is an instance of a VT.

A digital twin (DT) is an extension of VT with DS, such that not only the 3D visualisation and 3D motion of an object is in focus, but all process-based functionalities and interactions of and with the object(s) is represented, even when no direct 3D 


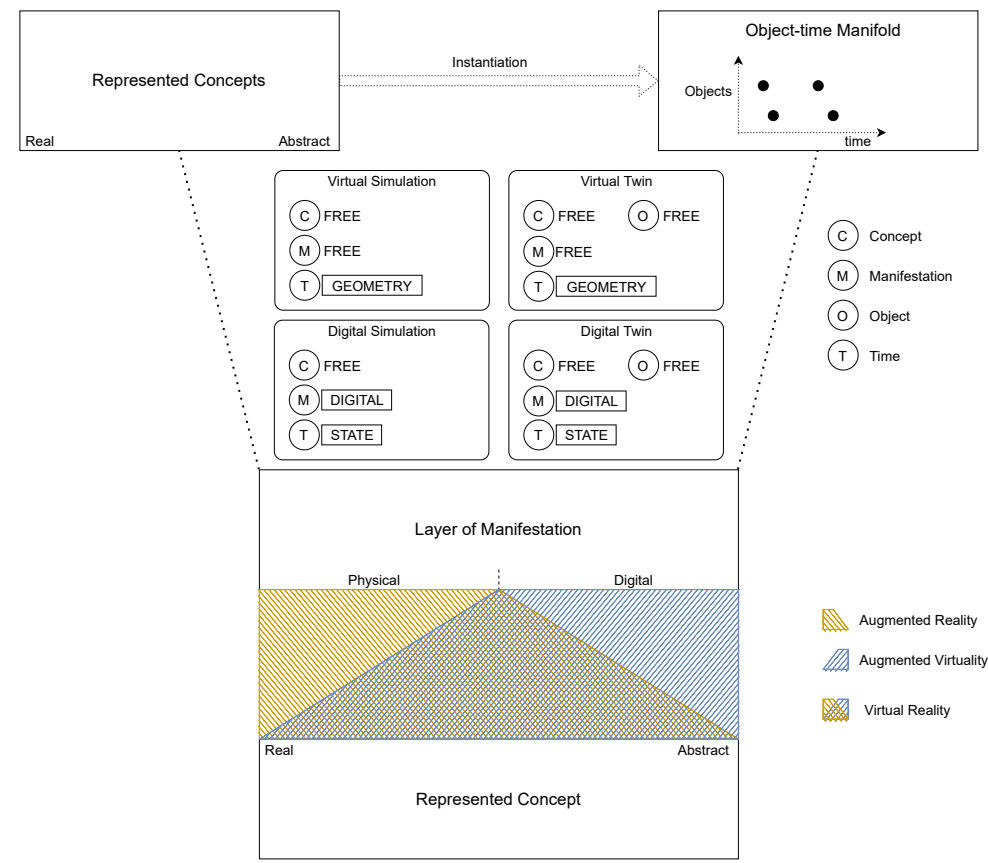

Figure 1

Key concepts introduced in Section 2. As the bottom of the figure shows, augmented reality augments a physical manifestation with concepts - possibly both real or abstract. Augmented virtuality on the other hand augments a digital manifestation with real or abstract concepts. Virtual reality is taken to integrate both of these possibilities, since the manifestation of VR can be both digital or physical (as in the case of an open-air historic replica of a bygone era). As the top of the figure shows, simulations and twins can be represented as a combination of concepts, manifestations, temporal representations and (in the case of twins) objects. The categories of virtual / digital and simulation / twin constrain some of these dimensions - i.e. virtual simulations / twins focus on geometry, while digital simulations / twins focus more on abstract state, and also, digital simulations / twins are constrained to the digital world, whereas virtual simulations / twins can be both physical and virtual.

geometrical representation for the given phenomenon exists. DT extends the concept of VT with user interactions and various further functionalities that are based on DS or obtained via a connection to the referent object. In general, if there are two objects, one being a real physical object and the other represented in VR, and the two are not only visually similar but can also be interacted with in similar ways and in synchrony, then the application is an instance of DT.

\subsection{Summary of Key Concepts}

To summarize, virtual, augmented and other mixed realities are arrangements of virtual objects, i.e. objects that in some way resemble a counterpart from a different 


\begin{tabular}{|c|c|c|c|c|c|}
\hline & \multirow{2}{*}{$\begin{array}{r}\text { Environment } \\
\text { Physical / Digital }\end{array}$} & \multirow{2}{*}{$\frac{\text { Symbols }}{\text { Physical / Digital }}$} & \multicolumn{3}{|c|}{ Referents } \\
\hline & & & $\begin{array}{l}\text { Geometric / } \\
\text { Abstract }\end{array}$ & $\begin{array}{l}\text { Static / } \\
\text { Temporal }\end{array}$ & $\begin{array}{l}\text { Real / } \\
\text { Imagined }\end{array}$ \\
\hline Virtual reality (VR) & $\mathrm{P} / \mathrm{D}$ & $\mathrm{P} / \mathrm{D}$ & G/A & $\mathrm{S} / \mathrm{T}$ & $\mathrm{R} / \mathrm{I}$ \\
\hline \multirow[t]{2}{*}{ Augmented reality (AR) } & $\mathrm{P}$ & $\mathrm{P} / \mathrm{D}$ & G/A & $\mathrm{S} / \mathrm{T}$ & $\mathrm{R} / \mathrm{I}$ \\
\hline & include as components: & & & & \\
\hline Virtual simulation (VS) & P/D & $\mathrm{P} / \mathrm{D}$ & G & $\mathrm{T}$ & $\mathrm{R} / \mathrm{I}$ \\
\hline Digital simulation (DS) & $\mathrm{D}$ & $\mathrm{D}$ & A & $\mathrm{T}$ & $\mathrm{R} / \mathrm{I}$ \\
\hline Virtual twin (VT) & $\mathrm{P} / \mathrm{D}$ & $\mathrm{P} / \mathrm{D}$ & G & $\mathrm{T}$ & $\mathrm{R}$ \\
\hline Digital twin (DT) & D & D & A & $\mathrm{T}$ & $\mathrm{R}$ \\
\hline
\end{tabular}

Table 1

Summary of concepts defined in Section 2. As indicated in the subheaders of the columns, the letters P, D, G, A, T, R, and I stand for Physical, Digital, Geometric, Abstract, Static, Temporal, Real and Imagined, respectively.

reality - whether physical, digital, real or imagined. The purpose of such realities can be, but is not necessarily to convey any immediate message about these counterparts; instead, their purpose can simply be to provide an aesthetically pleasing experience, or to serve as a medium (an environment) of communication through which other, related concepts can be conveyed more easily [17]. The main distinction between virtual and augmented reality is that an augmented reality is by necessity primarily physically based, with some (physical or digital) extensions. Even the physical or digital augmentation of the human body (viewed as a physical substrate) can be considered as an augmented reality, if it has the qualities of a reality as defined in Section 2.1.

Virtual and digital simulations, as well as virtual and digital twins can be seen as components that may be integrated into a virtual or augmented reality. At a conceptual level, virtual simulations and digital simulations can be defined as temporal representations of changes in the physical appearance, or in the state of some referent objects. Note that these referent objects need not actually exist in another reality (i.e. can be purely imagined), and the main focus is on temporal changes in the states of referent objects that may possibly exist.

Finally, we have defined virtual twins and digital twins as representations whose state evolves with the states of some referent objects which actually exist in a different reality. The purpose of such representations is to support the control and monitoring of systems that exist in a base reality that has a unique importance.

A summary of these concepts is provided in Table 1 and in Figure 1. 


\section{Definition of the Digital Reality}

\subsection{Deepening Pervasiveness of 2D Digital Environments}

The term 2D digital environment (2DE) refers to all software applications available to users on their infocommunication devices, i.e. any digital feature that provides 2-dimensional visual and interaction surfaces, including all web applications, 2D collaborative solutions and cloud based solutions that we use for work, entertainment and digital social life can be considered as 2D digital environments.

2DEs have become a central tool in the lives of most people living in the developed world for at least the past decade. However, the pervasiveness and influence of these environments is now deepening, as a result of a confluence of factors enabling automation at several different conceptual levels:

- Automation at the network level is leading to increased throughput and geographically more widespread availability, thereby enabling users to be presented with richer content in increasingly remote corners of the world.

- Automation dealing with big data solutions is allowing service providers to gather more information about and better understand the habits and preferences of their customers.

- Finally, artificial intelligence (AI) is making it possible to filter through this deluge of information and enables a more efficient curation of digital content in a context-sensitive way.

The net effect of these trends is not simply that humans are able to obtain the same information faster, nor simply that humans are able to obtain more information at any given time; but rather to release a catalytic process that is transforming our everyday reality - which is to say, our conception and perception of what is possible, desirable and achievable based on an integrated set of physical, digital and mental concepts.

Today, the World Wide Web is not just an Internet-based set of technologies that we use to access information and to send text, voice and / or video messages. Rather, we can increasingly communicate with machines directly and they are also capable of doing so with each other. Algorithms are 'watching over us', the big tech companies can easily learn things about us that we ourselves do not know. Further, they are able to obtain information that we willingly (or unknowingly) provide via a growing range of modalities. As non-human entities are entering the public Internet, and are evolving to a level where they can communicate, and eventually even decide / act on their own based on sophisticated adaptive methods, the associated cognitive load placed on humans is expected to be enormous [21]. 


\subsection{The Emergence of Cognitive Entities and Digital Reality}

When a human is growing up in such a complex digital environment his or her brain, mentality and complete psychological system is highly influenced (in many aspects irreversibly). In addition, the vast information that is collected and processed by AI algorithms is also qualitatively different compared to the past decades. In short, both humans' mental worlds and the digital world are influenced in a tight iteration loop. As a result, a markedly hybrid system is emerging in which the human and digital components are inseparable. This can be conceived of as the emergence of the Cognitive Entity, in which artificial and real cognitive systems are co-evolved to form qualitatively new capabilities $[7,22,23,24]$.

This view of humans will be even more relevant in the case of the coming generations. And it is based on this view, influenced by the concepts of VR, AR, VS, DS, VT, DT, AI and others in the field of cognitive infocommunications (CogInfoCom) $[7,6]$ that the concept of Digital Reality can be defined as follows:

A Digital Reality (DR) is a high-level integration of virtual reality (including augmented reality, virtual and digital simulations and twins), artificial intelligence and $2 D$ digital environments which creates a highly contextual reality for humans in which previously disparate realms of human experience are brought together. DR encompasses not only industrial applications but also helps increase productivity in all corners of life (both physical and digital), thereby enabling the development of new social entities and structures, such as $3 D$ digital universities, $3 D$ businesses, $3 D$ governance, $3 D$ web-based digital entertainment, $3 D$ collaborative sites and marketplaces.

A key question that may arise is how a discussion on Digital Reality differs from an analysis on "all digital solutions" or "everything that is digital". In our view, the term DR is more specific in that it refers to an integration of digital solutions that points to and as a result, helps create a new reality that involves human immersion (via co-evolution as discussed under Cognitive Infocommunications) and can be highly contextual.

As an example, consider an 'integration' of a physical refrigerator, microwave oven, smart stove and deep fryer along with their corresponding digital twins, together with various further virtual objects in a shareable VR space, which also includes a 2D digital environment of collaborative cookbooks, cooking notes, cooking blogs, and video chats with a connection to the most renowned experts in the culinary arts. In such an integration, with physical devices, functional replicas (for e.g. training and visualization), documentations and even access to the world's best cooks, the end result is a mixed reality that embodies a qualitatively more advanced reality than do its individual components - a digital reality for cooking. A DR like this might also include AI algorithms for making suggestions as the user is doing the cooking, or for aggregating the calories and nutritional value of the user's consumption over time, or for replenishing the supply of raw ingredients in a predictive manner. 
As demonstrated by this example, a digital reality should not be taken to mean everything that is digital; much rather, it defines a level of compactness, or concentratedness of components (whether physical, digital, static, dynamic, pre-programmed or AI-driven) that enables higher-level goals and functionalities to be formulated and implemented. This ability of DR to stretch even our mental concepts (of what is possible) and of extending our human capabilities in radically new ways is what sets it apart from mere collections of digital tools. Of course, DRs involve digital tools (among others), but those tools are organized and integrated in a specific, topic-oriented way. In short, DRs are capable of forming new realities analogous to what we mean by the term 'reality' as a web of concepts and experiences in terms of aspirations and actualization in real life.

\section{Internet of Digital Reality}

Based on the above, the Internet of Digital Reality (IoD) is a set of technologies that enables digital realities to be managed, transmitted and harmonized in networked environments (both public and private), focusing on a higher level of user accessibility, immersiveness and experience with the help of virtual reality and artificial intelligence. Connections among various cognitive entities also have to be handled not only at the end user level of virtual reality displays and software, but also at the levels of network protocols and network management, physical media (wired or wireless), hardware interfaces, and other equipment. AI is a key component of both digital reality and IoD, that enables a cohesion of context-driven content and intelligent network routing to emerge. In this section, we provide a brief overview of IoD. Further discussions of IoD, as well as its ramifications and the technologies behind it can be found in [11].

\subsection{Historical perspectives behind IoD}

The Internet of Things (IoT) introduced the world of networked "things" - e.g. sensors and actuators, wearables, digital twins - by integrating distributed computation with intelligent connections. Digital representations of physical entities in the real world can thus be connected, interacted with, managed, and they are able to communicate with each other even without constant human supervision.

The Internet of Everything (IoE) was defined by Cisco in 2013 as "bringing together people, process, data, and things to make networked connections more relevant and valuable than ever before, turning information into actions that create new capabilities, richer experiences, and unprecedented economic opportunity for businesses, individuals, and countries" [25, 26]. A key difference between IoE and IoT is the intelligence of connections. IoT is mostly about physical objects and concepts communicating with each other but IoE is what brings in network intelligence to bind all these concepts into a cohesive system. 
The proliferation of fields called 'Internet-of- $X$ ' (X being something different in each case, as in the case of e.g. Internet of Nano Things, Internet of MissionCritical Things, Internet of Mobile Things [27]) somewhat resembles the proliferation of more traditional fields focusing on different kinds of interactions - e.g. human-computer interactions, human-machine interactions, human-robot interactions etc. The emergence of such fields with similar concepts and methodologies (albeit applied to different contexts) was part of the impetus behind the definition of cognitive infocommunications (CogInfoCom) [6, 7, 24]. Similarly, we propose the term 'Internet of Digital Reality (IoD)' to partially integrate, partially complement and more importantly augment earlier notions of the form 'Internet-of-X'. After all, it can be argued that the real motivation behind all of these technological directions was always to merge, augment and share realities - an idea that is already present (excluding the network aspects) in Digital Reality.

Perhaps the most relevant aspect of IoD is that it connects digital realities, that is, combinations of technologies and data that create a higher-level functional integration. For example, IoD is the network through which combinations of $3 \mathrm{D}$ virtual spaces and their real-world counterparts can be shared, together with all relevant data and interactive support for extended capabilities.

\subsection{Pillars of IoD}

In one sense, IoD supersedes IoT and IoE where not only physical "things", but also complete digital reality are connected via a (public or private) network.

Today, cognitive entities and virtual / digital twins are strongly based on Internet connections [28]. Pillars of the Internet of Digital Reality include:

- Cognitive entities (qualitatively new capabilities emerging via a combination of machines, "things", sensors, AI, digital twins, avatars, algorithms, bots, RPA, higher level organizations etc.) interacting in the digital realm

- Information (data, web content, control)

- Communication networks (intelligent connection, wired and RF physical layers, public and private networks)

- Artificial intelligence, which gains new significance as a global network capable of distributed learning and continued evolution as digital realities become connected and shared across the globe

- Access devices and interfaces (headsets, tactile devices, AR/MR/VR spaces, 360-degree immersive scenarios, mobility and navigation)

- Cognitive infocommunications (sensation and perception, human factors and human-ICT co-evolution) 
- Safety and security (data, information, or even the physical safety of the user etc.)

- Digital business and legal issues

- Digital Society (education, acceptance of technology, digital work, e-government, digital arts and gaming)

All of the above pillars are a part of the science and practice of IoD; however, these pillars are also broadened in scope based on the radically new perspectives of IoD. For example, artificial intelligence is transformed into a global network (networked $\mathrm{AI})$ that is capable of learning based on user activities and user data on the fly. Of course, the breaking down of barriers between various contexts and use cases also creates new challenges in terms of data security and legal issues. These are just two examples of how the pillars of IoD both help form and are transformed by IoD. A detailed discussion of IoD, as well as its ramifications and the technologies behind it can be found in Part II of this paper [11].

\section{Conclusions}

In this paper, a short introduction was given on the concept of Digital Reality, which allows users to access, manipulate and interact with integrated sets of content - whether on display screens, immersive settings or overlaid on physical objects that represent concepts and / or objects that can be both physical or digital, as well as both real or imagined. The paper showed that this new form of reality born in today's high level entangled combination of digital technologies and humans needs a new definition for future scientific works. Further, the concept of Internet of Digital Reality (IoD) was introduced, as a set of network and related technologies for the management, transition and harmonization of Digital Realities. Further details on IoD can be found in [11].

\section{Acknowledgement}

This research was supported by the Digital Development Center in the national framework GINOP-3.1.1-VEKOP-15- 2016-00001 "Promotion and support of cooperations between educational institutions and ICT enterprises".

\section{References}

[1] K. K. Loh and R. Kanai, "How has the Internet Reshaped Human Cognition?" The Neuroscientist, vol. 22, no. 5, pp. 506-520, 2016.

[2] M. Prensky, "Homo Sapiens Digital: From Digital Immigrants and Digital Natives to Digital Wisdom," Innovate: Journal of Online Education, vol. 5, no. 3, 2009.

[3] C. Frauenberger, "Entanglement HCI the Next Wave?" ACM Transactions on Computer-Human Interaction (TOCHI), vol. 27, no. 1, pp. 1-27, 2019. 
[4] R. Kurzweil, The singularity is near: When humans transcend biology. Penguin, 2005.

[5] A. Pilsch, Transhumanism: Evolutionary Futurism and the Human Technologies of Utopia. U of Minnesota Press, 2017.

[6] P. Baranyi and Á. Csapó, "Definition and synergies of cognitive infocommunications," Acta Polytechnica Hungarica, vol. 9, no. 1, pp. 67-83, 2012.

[7] P. Baranyi, A. Csapo, and G. Sallai, Cognitive Infocommunications (CogInfoCom). Springer, 2015 .

[8] P. D. Ramani Moses, Nikita Garia, "Digital Reality - A technical primer," https://www2.deloitte.com/insights/us/en/topics/emerging-technologies/ digital-reality-technical-primer.html, 2021.

[9] J. Schwartz, S. Hatfield, R. Jones, and S. Anderson, "What is the Future of Work," Redefining work, workforces, and workplaces. Part Of A Deloitte Series On The Future Of Work, 2019.

[10] Y. Kang and K. C. Yang, "Employing Digital Reality Technologies in Art Exhibitions and Museums: A Global Survey of Best Practices and Implications," in Virtual and Augmented Reality in Education, Art, and Museums. IGI Global, 2020, pp. 139-161.

[11] G. Wersényi, Á. Csapó, T. Budai, and P. Baranyi, "Internet of Digital Reality: Infrastructural Background - Part II," Acta Polytechnica Hungarica, vol. 18, no. 8, pp. 91-104, 2021.

[12] H. Ishii, "The tangible user interface and its evolution," Communications of the $A C M$, vol. 51, no. 6, pp. 32-36, 2008.

[13] Y. Zhao, Y. Qin, Y. Liu, S. Liu, and Y. Shi, "Qook: A new physical-virtual coupling experience for active reading," in Proceedings of the adjunct publication of the 26th annual ACM symposium on User interface software and technology, 2013, pp. 5-6.

[14] A. M. Glenberg, "Few believe the world is flat: How embodiment is changing the scientific understanding of cognition." Canadian Journal of Experimental Psychology/Revue canadienne de psychologie expérimentale, vol. 69, no. 2, p. $165,2015$.

[15] L. W. Barsalou et al., "Perceptual symbol systems," Behavioral and brain sciences, vol. 22, no. 4, pp. 577-660, 1999.

[16] B. Z. Mahon and A. Caramazza, "A critical look at the embodied cognition hypothesis and a new proposal for grounding conceptual content," Journal of physiology-Paris, vol. 102, no. 1-3, pp. 59-70, 2008. 
[17] Á. B. Csapó, I. Horvath, P. Galambos, and P. Baranyi, "VR as a medium of communication: from memory palaces to comprehensive memory management," in 2018 9th IEEE International Conference on Cognitive Infocommunications (CogInfoCom). IEEE, 2018, pp. 000389-000 394.

[18] P. Milgram, H. Takemura, A. Utsumi, and F. Kishino, "Augmented Reality: A Class of Displays on the Reality-Virtuality Continuum," in Telemanipulator and telepresence technologies, vol. 2351. International Society for Optics and Photonics, 1995, pp. 282-292.

[19] R. J. Grossmann, "Virtual reality, Augmented Reality - I call it i-Reality," Mhealth, vol. 1, 2015.

[20] D. Williams, "The Internet of Everything - Cisco IoE Value Index Study," https://www.huffpost.com/entry/the-history-of-augmented-_b_9955048, 2017.

[21] F. Pasquale, "The alien intelligence of automated media," in New Laws of Robotics. Harvard University Press, 2020, pp. 89-118.

[22] P. Baranyi and A. B. Csapo, "Revisiting the concept of generation CEGeneration of Cognitive Entities," in 2015 6th IEEE International Conference on Cognitive Infocommunications (CogInfoCom). IEEE, 2015, pp. 583-586.

[23] L. I. Komlósi and P. Waldbuesser, "The cognitive entity generation: Emergent properties in social cognition," in 2015 6th IEEE International Conference on Cognitive Infocommunications (CogInfoCom). IEEE, 2015, pp. 439-442.

[24] J. Katona, "A Review of Human-Computer Interaction and Virtual Reality Research Fields in Cognitive InfoCommunications," Applied Sciences, vol. 11, no. 6 , p. 2646, 2021.

[25] Cisco, "The Internet of Everything - Global Private Sector Economic Analysis," https://www.cisco.com/c/dam/en_us/about/ac79/docs/innov/IoE_Economy_ FAQ.pdf, 2013.

[26] _ _ "The Internet of Everything - Cisco IoE Value Index Study," https://www. cisco.com/c/dam/en_us/about/business-insights/docs/ioe-value-index-faq.pdf, 2013.

[27] C. Srinivasan, B. Rajesh, P. Saikalyan, K. Premsagar, and E. S. Yadav, "A Review on the Different Types of Internet of Things (IoT)," Journal of Advanced Research in Dynamical and Control Systems, vol. 11, no. 1, pp. 154-158, 2019.

[28] D. Jones, C. Snider, A. Nassehi, J. Yon, and B. Hicks, "Characterising the Digital Twin: A systematic literature review," CIRP Journal of Manufacturing Science and Technology, vol. 29, pp. 36-52, 2020. 